\title{
Tensor Analyzing Powers for Quasielastic Electron Scattering from Deuterium
}

\author{
Z.-L. Zhou, ${ }^{1 *}$ M. Bouwhuis, ${ }^{2}$ M. Ferro-Luzzi, ${ }^{2,3}$ E. Passchier, ${ }^{2}$ R. Alarcon, ${ }^{4}$ M. Anghinolfi, ${ }^{5}$ H. Arenhövel, ${ }^{6}$ \\ R. van Bommel, ${ }^{2}$ T. Botto, ${ }^{2}$ J. F. J. van den Brand, ${ }^{1,7}$ H. J. Bulten, ${ }^{1,7}$ S. Choi, ${ }^{4}$ J. Comfort, ${ }^{4}$ S. M. Dolfini, ${ }^{4}$ R. Ent, ${ }^{8}$ \\ C. Gaulard, ${ }^{4}$ D. W. Higinbotham, ${ }^{9}$ C. W. de Jager, ${ }^{2, \dagger}$ E. Konstantinov, ${ }^{10}$ J. Lang, ${ }^{3}$ W. Leidemann, ${ }^{11}$ D. J. de Lange, ${ }^{2}$ \\ M. A. Miller, ${ }^{1, \dagger}$ D. Nikolenko, ${ }^{10}$ N. Papadakis, ${ }^{2}$ I. Passchier,${ }^{2}$ H. R. Poolman, ${ }^{2}$ S. G. Popov, ${ }^{10,8}$ I. Rachek, ${ }^{10}$ M. Ripani, ${ }^{5}$ \\ E. Six, ${ }^{4}$ J. J. M. Steijger, ${ }^{2}$ M. Taiuti, ${ }^{5}$ O. Unal, ${ }^{1}$ N. Vodinas,${ }^{2}$ and H. de Vries ${ }^{2}$ \\ ${ }^{1}$ Department of Physics, University of Wisconsin, Madison, Wisconsin 53706 \\ ${ }^{2}$ NIKHEF, P.O. Box 41882, NL-1009 DB Amsterdam, The Netherlands \\ ${ }^{3}$ Institut für Teilchenphysik, Eidgenössische Technische Hochschule, CH-8093 Zürich, Switzerland \\ ${ }^{4}$ Department of Physics, Arizona State University, Tempe, Arizona 85287 \\ ${ }^{5}$ Istituto Nazionale di Fisica Nucleare (INFN), I-16146 Genova, Italy \\ ${ }^{6}$ Institut für Kernphysik, Johannes Gutenberg Universität, D-55099 Mainz, Germany \\ ${ }^{7}$ Department of Physics and Astronomy, Vrije Universiteit, NL-1081 HV Amsterdam, The Netherlands \\ ${ }^{8}$ TJNAF, Newport News, Virginia 23606, \\ and Department of Physics, Hampton University, Hampton, Virginia 23668 \\ ${ }^{9}$ Department of Physics, University of Virginia, Charlottesville, Virginia 22901 \\ ${ }^{10}$ Budker Institute for Nuclear Physics, Novosibirsk, 630090 Russian Federation \\ ${ }^{11}$ Dipartimento di Fisica and INFN (gruppo collegato di Trento), Università di Trento, I-38050 Povo (Trento), Italy
}

(Received 18 September 1998)

We report on a first measurement of tensor analyzing powers in quasielastic electron-deuteron scattering at an average three-momentum transfer of $1.7 \mathrm{fm}^{-1}$. Data sensitive to the spin-dependent nucleon density in the deuteron were obtained for missing momenta up to $150 \mathrm{MeV} / c$ with a tensor polarized ${ }^{2} \mathrm{H}$ target internal to an electron storage ring. The data are well described by a calculation that includes the effects of final-state interaction, meson-exchange and isobar currents, and leading-order relativistic contributions. [S0031-9007(98)08241-6]

PACS numbers: 25.30.Fj, 21.45.+v, 24.70.+s, 29.25.Pj

The deuteron is often used as a benchmark to test nuclear theory, since reliable calculations can be performed in both nonrelativistic and relativistic models. Observables such as its binding energy, static magnetic dipole and charge quadrupole moment, asymptotic $D / S$ ratio, and the elastic electromagnetic form factors place strong constraints on models for the nuclear interaction. Furthermore, the deuteron is one of the few nuclear systems for which predictions based on quantum chromodynamics have been made and tested [1-4]. The understanding of the deuteron has progressed to a level which allows the deuteron to be used as an effective neutron target in studies of the electromagnetic form factors of the neutron [5-8] and the spin structure functions of the neutron in deep inelastic scattering [9-11].

Quasielastic scattering has been applied to determine the momentum distribution of the proton in the deuteron [12] with necessary corrections for the interaction effects [13] and to study the $\left(e, e^{\prime} p\right)$ reaction mechanism itself [14]: the necessity of including relativistic corrections has been demonstrated by determining the longitudinaltransverse interference structure function; details of the electromagnetic coupling to the nuclear currents have been studied by performing longitudinal-transverse separations and polarization transfer measurements, and the influence of final-state interaction (FSI) effects has been isolated by studying the fifth structure function. However, all of these measurements were performed with unpolarized deuterons. The use of polarized deuterium is advantageous, since one can select polarization observables that are sensitive to small amplitudes that are related to, for example, the charge form factor of the neutron, or the $D$ wave admixture in the ground-state wave function [15]. Here, we report on the first measurement of quasielastic electron scattering from polarized deuterium.

It is well known that the tensor force has an important influence on deuteron structure. It introduces $D$-state components into the predominantly $S$-wave ground-state wave function and hence leads to a density distribution that depends on the spin projection $m_{z}$. Because of the tensor part of the nucleon-nucleon interaction and the repulsive core at short distances, the deuteron exhibits a toroidal shape for $m_{z}=0$ and a dumbbell shape for $m_{z}=$ \pm 1 (see, e.g., [16]). The spin-dependent momentum density distribution $\rho_{m_{z}}$ can be written as

$$
\begin{aligned}
\frac{\rho_{0}(\mathbf{p})}{4 \pi} & =\left[R_{0}+\sqrt{2} R_{2} d_{0,0}^{2}(\theta)\right]^{2}+3\left[R_{2} d_{1,0}^{2}(\theta)\right]^{2}, \\
\frac{\rho_{ \pm 1}(\mathbf{p})}{4 \pi} & =\left[R_{0}-\frac{1}{\sqrt{2}} R_{2} d_{0,0}^{2}(\theta)\right]^{2}+\frac{9}{8} R_{2}^{2}\left(1-\cos ^{4} \theta\right),
\end{aligned}
$$

with $R_{0}(p)$ and $R_{2}(p)$ the usual radial wave functions for orbital angular momentum $L=0$ and $L=2$ in momentum space, respectively, $d_{m, m^{\prime}}^{j}$ the rotation functions, and 
$\theta$ the angle between the polarization axis and the relative momentum $\mathbf{p}$ of the two nucleons.

The spin-dependent density distribution can be probed by quasielastic electron scattering from tensor-polarized deuterium targets, which yields data complementary to the elastic channel [17-19] where the total nuclear current (i.e., nucleon and meson contributions) is probed. In plane-wave impulse approximation (PWIA), the $\left(e, e^{\prime} p\right)$ cross section for unpolarized electrons factorizes in a part depending on the off-shell electron-proton cross section and a part containing the spin-dependent momentum distribution as shown in Eq. (1) [20]. In that case the relative nucleon momentum $\mathbf{p}$ can be related to the missing momentum of the reaction, defined as $\mathbf{p}_{\mathrm{m}}=\mathbf{q}-\mathbf{p}^{\prime}$, with $\mathbf{q}$ the momentum transferred in the electron scattering process and $\mathbf{p}^{\prime}$ the momentum of the knocked-out proton. In a cross section measurement with tensor-polarized deuterons one can define the tensor analyzing power $A_{d}^{T}$ :

$$
\begin{gathered}
A_{d}^{T}=\sqrt{\frac{1}{2}} \frac{\sigma_{+}\left(p_{m}\right)+\sigma_{-}\left(p_{m}\right)-2 \sigma_{0}\left(p_{m}\right)}{\sigma_{+}\left(p_{m}\right)+\sigma_{-}\left(p_{m}\right)+\sigma_{0}\left(p_{m}\right)} \\
\stackrel{\text { PWIA }}{=}-\frac{2 R_{0}(p) R_{2}(p)+\sqrt{\frac{1}{2}} R_{2}^{2}(p)}{R_{0}^{2}(p)+R_{2}^{2}(p)} d_{0,0}^{2}(\theta),
\end{gathered}
$$

with $\sigma_{0}\left(\sigma_{ \pm}\right)$the cross section measured with $m_{z}=0$ $\left(m_{z}= \pm 1\right)$ and $d_{0,0}^{2}=\frac{3}{2} \cos ^{2} \theta-\frac{1}{2}$. In the limit where $R_{2} \ll R_{0}, A_{d}^{T}$ directly relates to the ratio $R_{2} / R_{0}$. Furthermore, in PWIA when $R_{0}=-\sqrt{2} R_{2}$, then $\sigma_{0}=0$ and $A_{d}^{T}$ is maximum with a value of $+\sqrt{\frac{1}{2}}$ for $\theta=0$. Even when FSI and all other effects are included, the cross section is expected to be small for electron scattering from deuterons with $m_{z}=0$. Similarly, when $R_{0}=\sqrt{\frac{1}{2}} R_{2}$, the PWIA cross section for deuterons with $m_{z}= \pm 1$ vanishes and $A_{d}^{T}$ reaches a minimum of $-\sqrt{2}$.

The experiment was performed with a polarized gas target [21] internal to the AmPS electron storage ring at NIKHEF. A beam current of $120 \mathrm{~mA}$ was injected. The $565 \mathrm{MeV}$ electron beam had a lifetime of about $15 \mathrm{~min}$. An atomic beam source was used to inject a flux of $1.1 \times 10^{16}$ atoms/s with two hyperfine states into a $T$-shaped storage cell, which was cooled to $100 \mathrm{~K}$ in order to further increase the target density. The data were obtained with Teflon-coated aluminum cells (wall thickness $25 \mu \mathrm{m}$ ) with diameters of 15 or $20 \mathrm{~mm}$ and a length of $400 \mathrm{~mm}$. The resulting target thickness amounted up to $2 \times 10^{13}\left({ }^{2} \mathrm{H}\right.$ atoms $) / \mathrm{cm}^{2}$. The tensor polarization of the target $P_{\mathrm{zz}}\left(=1-3 n_{0}\right.$, with $n_{0}$ the fraction of deuterons with $m_{z}=0$ ) was varied every $10 \mathrm{~s}$ between $P_{\mathrm{zz}}^{+}=+0.488 \pm 0.014 \pm 0.03$ and $P_{\mathrm{zz}}^{-}=$ $-0.893 \pm 0.027 \pm 0.052$, where the first (second) error represents the statistical (systematic) uncertainty. The tensor polarization of the deuterium atoms was measured in situ by a polarimeter [22] that analyzes the fraction of the target gas that has been ionized by the traversing electron beam. Two electromagnets were used to generate a magnetic field in the interaction region that allows one to orient the target polarization axis in the electron scattering plane, either parallel or perpendicular to the momentum transfer. A set of scrapers was employed to intercept the halo of the electron beam and thus reduce the amount of background from the cell wall.

The experimental setup has been described in detail in Refs. [21,23], and results for elastic electron-deuteron scattering have been presented previously [18]; here only a brief overview is given. Electrons scattered from the tensor-polarized deuterium gas were detected in an electromagnetic calorimeter, consisting of six layers of CsI(Tl) blocks covering a solid angle of $180 \mathrm{msr}$. Two plastic scintillators, one in front of the $\mathrm{CsI}(\mathrm{Tl})$ blocks and one positioned between the first two layers, provided the electron trigger. The total energy resolution obtained (about $22 \mathrm{MeV}$ ) was sufficient to distinguish between events from quasielastic scattering and events from pion electroproduction. Two sets of wire chambers, one adjacent to the scattering chamber and one in front of the first trigger scintillator, were used for track reconstruction. The central angle of the electron detector was $35^{\circ}$ corresponding to an average transferred three momentum of $|\mathbf{q}| \approx 1.7 \mathrm{fm}^{-1}$.

The ejected protons were detected in a range telescope, consisting of 15 layers of $1 \mathrm{~cm}$ thick plastic scintillator preceded by a layer of $2 \mathrm{~mm}$ thick plastic scintillator. The trigger was formed by a coincidence between the first two layers. The detector was positioned at a central angle of $80^{\circ}$ and covered a solid angle of nearly $300 \mathrm{msr}$. The range telescope was preceded by two wire chambers for track reconstruction. Protons in the range of 30$100 \mathrm{MeV}$ were detected with an energy resolution of about $1.5 \mathrm{MeV}$.

Calibration measurements were performed with the kinematically overdetermined reaction ${ }^{1} \mathrm{H}\left(e, e^{\prime} p\right)$. Since the calorimeter excludes the pion-production channels, the fivefold differential cross section can be obtained by measuring the electron scattering angles $\theta_{e}, \phi_{e}$, the angles of the ejected proton $\theta_{p}, \phi_{p}$, and the energy of the proton. This procedure resulted in an excellent missing-momentum resolution, for a nonmagnetic detector setup, of $6.2 \mathrm{MeV} / c$ as demonstrated in Fig. 1 (top panel). In the bottom panel of the figure, the missingmomentum spectrum for scattering from deuterium is shown. The data are in reasonable agreement with the result of a Monte Carlo calculation that includes the offshell electron-proton cross section of de Forest, Jr. [24], momentum densities from Bernheim et al. [12], and a model for the detector phase space. The shaded histogram represents the background contribution which was determined by scattering from an empty cell. It is seen that the background contributes significantly to the data taken for missing momenta above $150 \mathrm{MeV} / c$.

Figure 2 shows the measured asymmetry, $A_{d}^{T}$, as a function of the angle $\theta_{s}$ between the polarization axis and 


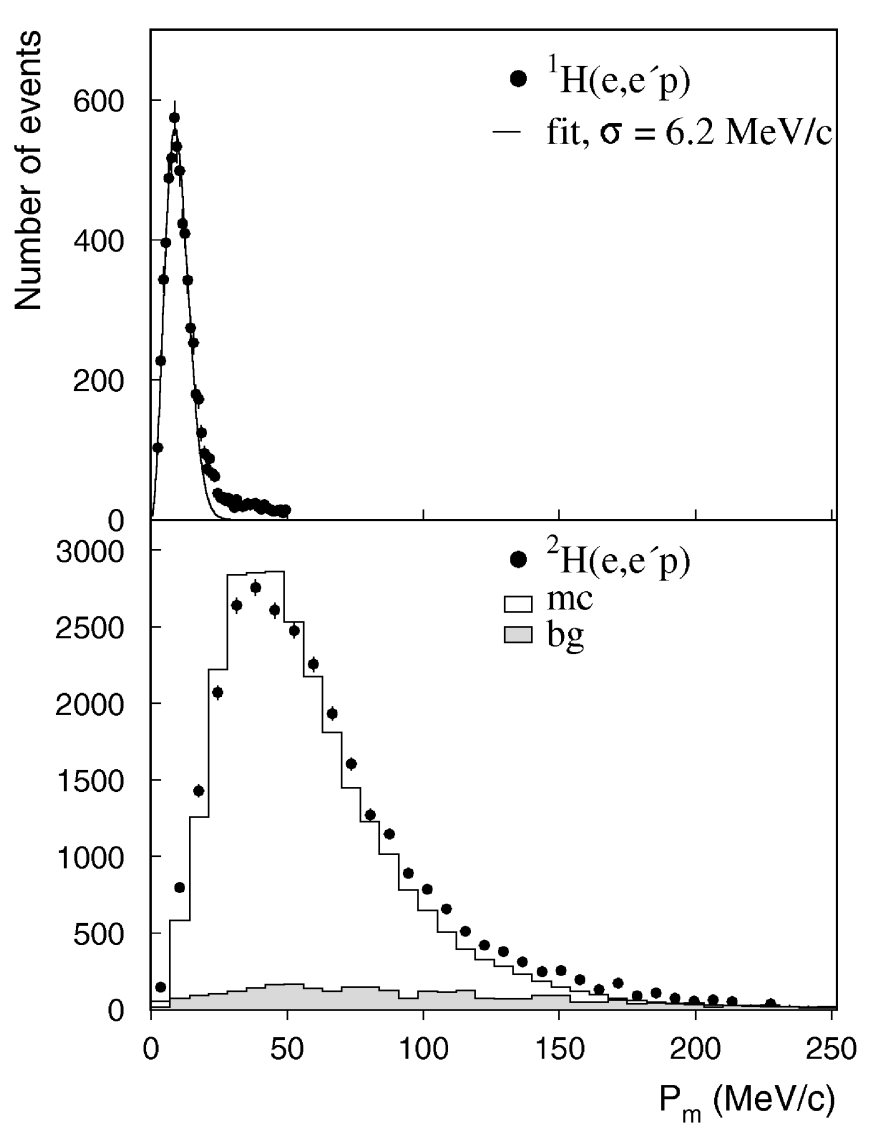

FIG. 1. Top panel: Missing-momentum spectrum for the kinematically overcomplete reaction ${ }^{1} \mathrm{H}\left(e, e^{\prime} p\right)$. Bottom panel: Missing-momentum spectrum for scattering from deuterium. The shaded histogram indicates the background from the cell wall. The solid histogram shows a Monte Carlo prediction.

the missing momentum. Results of measurements with the polarization axis parallel and perpendicular to the momentum transfer were combined in this figure. The data are compared to predictions [15] for the Paris potential. The dashed curve in Fig. 2 represents the results for the plane-wave Born approximation (PWBA) which includes the coupling to the neutron, whereas in the solid curve the effects of final-state interaction, meson-exchange (MEC) and $\Delta$-isobar (IC) currents, and relativistic corrections are included. The spread in kinematics in each bin has been taken into account by applying a Monte Carlo code that interpolated between a dense grid of calculations that covered the full acceptance of our setup. It is observed that the calculations describe the data well. Since in PWIA the asymmetry is proportional to $d_{0,0}^{2}$ [see Eq. (2)], the zero crossings of the asymmetry are predicted to be at $\cos \theta_{s}= \pm \sqrt{\frac{1}{3}}(\approx 0.58)$, while without a $D$-state component to the ground-state wave function the asymmetry vanishes. The results for PWBA differ slightly from this prediction, indicating that for our kinematics the coupling to the neutron is not completely negligible. Furthermore, it is demonstrated that FSI effects modify the asymmetry mainly for positive values of $\cos \theta_{s}$.

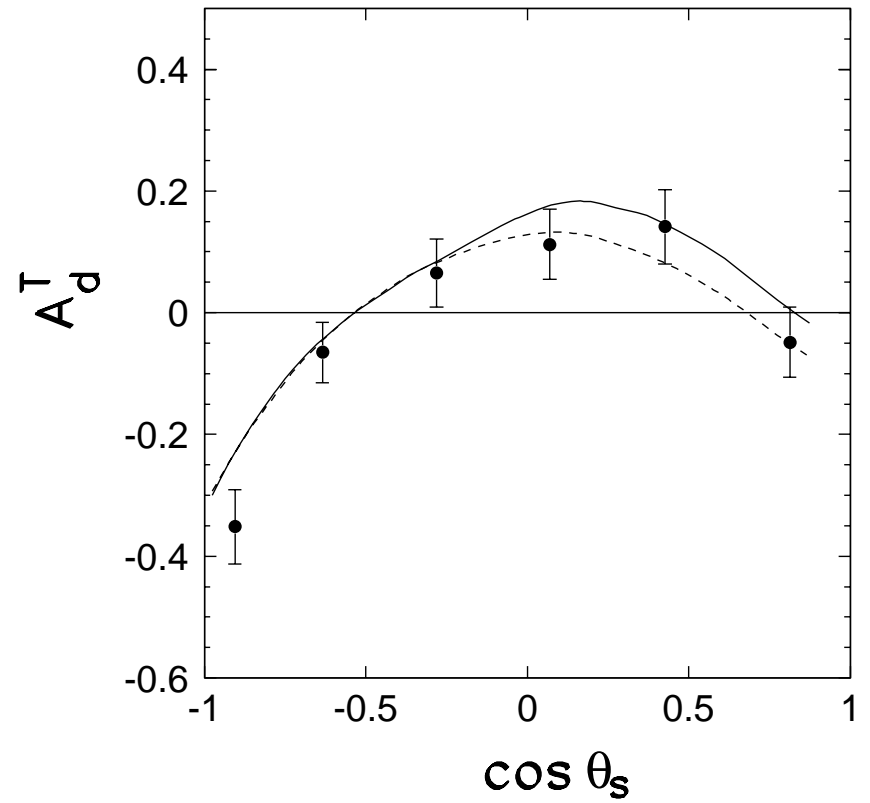

FIG. 2. $A_{d}^{T}$ as a function of $\cos \theta_{s}$. The dashed curve represents the result for PWBA, the solid curve for the full calculation including FSI, MEC, and IC contributions and relativistic corrections.

Figure 3 shows $A_{d}^{T}$ as a function of missing momentum in approximately parallel kinematics, i.e., the center-ofmass angle between $\mathbf{p}^{\prime}$ and $\mathbf{q}$ has been restricted $\left(\theta_{p q}^{\mathrm{cm}}<\right.$ $13^{\circ}$ ). The results of the full calculation describe the data well. The inclusion of spin-dependent rescattering effects improves the description at the lowest missing momenta

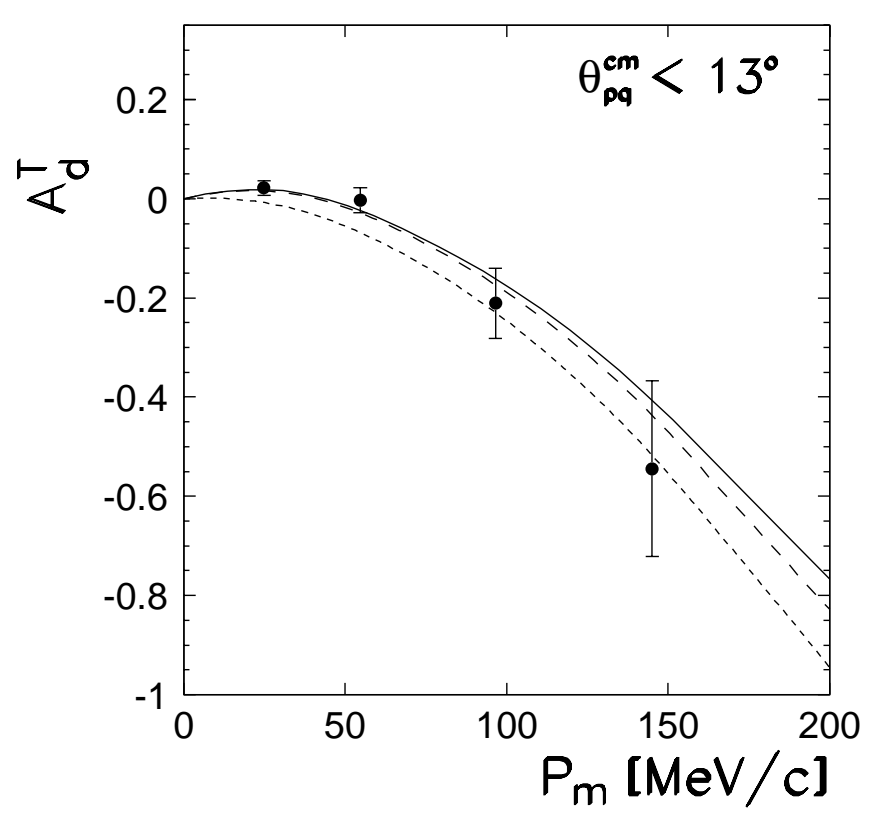

FIG. 3. $A_{d}^{T}$ as a function of $p_{m}$ for parallel kinematics (i.e., $\left.\theta_{p q}^{\mathrm{cm}}<13^{\circ}\right)$. The short-dashed curve represents the result for PWBA; in the long-dashed curve FSI effects are also included, and the solid curve represents the full calculation. 
$\left(p_{m} \simeq 50 \mathrm{MeV} / c\right)$, where $D$-state contributions are small. Note that the tensor analyzing power is sizable, even at the relatively small missing momenta addressed in the present experiment. This is due to the fact that an interference between the small $D$-state amplitude and the dominant $S$-state amplitude is probed [see Eq. (2)], whereas in the unpolarized cross section only the sum of the squares of the $S$-state and $D$-state amplitudes enter. Note that since the statistical uncertainties dominate, only those are shown in both Figs. 2 and 3.

We have investigated the influence of the choice of nucleon-nucleon potential on the calculated tensor analyzing powers. The results for $A_{d}^{T}$ calculated with the Paris, Bonn, Nijmegen, and Argonne potentials differ by less than $1 \%$ for missing momenta below $150 \mathrm{MeV} / c$ and therefore do not influence our conclusions. In addition, we studied the influence of relativistic effects in the current operator. $A_{d}^{T}$ changes by less than $2 \%$ when the model is modified to not include third- and fourth-order terms in $p / M_{p}$ in the current operator and to neglect contributions from the boost operator that translates the initial-state wave function from the laboratory frame to the center-of-mass frame.

In summary, a polarized deuterium target has successfully been utilized in quasielastic electron scattering. Tensor analyzing powers have been measured for missing momenta up to $150 \mathrm{MeV} / c$. The data are well described by the present model [15], using the Paris potential, and the tensor analyzing power is dominated by the effects of the $D$-state contribution. We have shown that the internal-target technique allows for novel measurements of quasifree scattering from the deuteron, which in the future should be extended to higher missing momenta, in the region where the tensor analyzing powers are predicted to strongly depend on the details of the deuteron spin structure $[16,25,26]$.

We gratefully acknowledge discussions with S. Jeschonnek and T.W. Donnelly on factorization issues of the spin-dependent $\left(e, e^{\prime} p\right)$ cross section. This work was supported in part by the Stichting voor Fundamenteel Onderzoek der Materie (FOM), which is financially supported by the Nederlandse Organisatie voor Wetenschappelijk Onderzoek (NWO), the Swiss National Foundation, the National Science Foundation under Grants No. PHY-9316221 (Wisconsin), No. PHY-9200435 (Arizona State), and No. HRD-9154080 (Hampton), NWO
Grant No. 713-119, and HCM Grants No. ERBCHBICT930606 and No. ERB4001GT931472.

*Present address: Laboratory for Nuclear Science, Massachusetts Institute of Technology, Cambridge, MA 02139.

†Present address: TJNAF, Newport News, VA 23606.

*Present address: University of Illinois, UrbanaChampaign, IL 61801.

${ }^{\S}$ Deceased.

[1] S. J. Brodsky and G. L. Farrar, Phys. Rev. Lett. 31, 1153 (1973).

[2] J. E. Belz et al., Phys. Rev. Lett. 74, 646 (1995).

[3] C. E. Carlson, Nucl. Phys. A508, 481c (1990).

[4] C. Bochna et al., Phys. Rev. Lett. 81, 4533 (1998).

[5] S. Platchkov et al., Nucl. Phys. A510, 740 (1990).

[6] T. Eden et al., Phys. Rev. C 50, R1749 (1994).

[7] F. Klein et al., Nucl. Phys. A623, 323c (1997).

[8] E. E. W. Bruins et al., Phys. Rev. Lett. 75, 21 (1995).

[9] B. Adeva et al., Phys. Lett. B 302, 533 (1993).

[10] K. Abe et al., Phys. Rev. Lett. 75, 25 (1995).

[11] HERMES Collaboration, K. Ackerstaff et al., DESY Report No. DESY-PRC-93-06, 1993 (unpublished).

[12] M. Bernheim et al., Nucl. Phys. A365, 349 (1981).

[13] H. Arenhövel, Nucl. Phys. A384, 287 (1982).

[14] S. Gilad, W. Bertozzi, and Z.-L. Zhou, Nucl. Phys. A631, 276c (1998), and references therein.

[15] H. Arenhövel, W. Leidemann, and E. L. Tomusiak, Phys. Rev. C 52, 1232 (1995).

[16] J. L. Forest et al., Phys. Rev. C 54, 646 (1996).

[17] I. The et al., Phys. Rev. Lett. 67, 173 (1991).

[18] M. Ferro-Luzzi et al., Phys. Rev. Lett. 77, 2630 (1996).

[19] TJNAF experiment E94-018, S. Kox and E.J. Beise, spokespersons.

[20] J. A. Caballero, T. W. Donnelly, and G. I. Poulis, Nucl. Phys. A555, 709 (1993).

[21] Z.-L. Zhou et al., Nucl. Instrum. Methods. Phys. Res., Sect. A 378, 40 (1996).

[22] Z.-L. Zhou et al., Nucl. Instrum. Methods. Phys. Res., Sect. A 379, 211 (1996).

[23] E. Passchier et al., Nucl. Instrum. Methods. Phys. Res., Sect. A 387, 471 (1997).

[24] T. de Forest, Jr., Nucl. Phys. A392, 232 (1983).

[25] L. L. Frankfurt and M. I. Strikman, Nucl. Phys. A405, 557 (1983).

[26] A. Bianconi, S. Jeschonnek, N. N. Nikolaev, and B. G. Zakharov, Phys. Rev. C 53, 576 (1996). 